\title{
Microscale Repeatability of the Shape-Memory Effect in Fine NiTi Wires
}

\author{
Joyce Yue Gong ${ }^{1} \cdot$ Samantha H. Daly ${ }^{2}$
}

Published online: 9 November 2016

(C) ASM International 2016

\begin{abstract}
An experimental investigation into microscale transformation characteristics of polycrystalline NiTi wires of $500 \mu \mathrm{m}$ diameter during shape memory cycling is discussed, with emphasis on the characterization of a pronounced heterogeneity in the strain distribution evident during detwinning of the martensite phase upon application of load and its persistence throughout the actuation cycle. Using scanning electron microscopy-digital image correlation, full-field strain maps at the microscale were obtained during shape memory cycling. It was found that the strains induced by detwinning were quite heterogeneous at the microscale, and could display a large degree of similarity with thermo-mechanical cycling that tended to increase as cycling progressed. Residual strain concentrated at locations where strain accumulation from detwinning and plasticity were significant, indicating that martensitic detwinning and the associated plasticity that occurs with it is spatially correlated to the subsequent accumulation of residual strain at the microscale.
\end{abstract}

Keywords NiTi $<$ materials . Shape memory - SMA · SEM-DIC $\cdot$ Thermo-mechanical cycling $\cdot$ Shape-memory effect

Samantha H. Daly

samdaly@engineering.ucsb.edu

1 Department of Mechanical Engineering, University of Michigan, 2350 Hayward Street, Ann Arbor, MI 48109, USA

2 Department of Mechanical Engineering, University of California at Santa Barbara, Engineering II, Santa Barbara, CA 93106, USA

\section{Introduction}

Shape-memory alloys (SMAs) refer to a class of metallic alloys that demonstrate unique properties including the shape-memory effect (SME) and superelasticity (SE). Both of these properties are achieved through a solid-to-solid, diffusionless phase transformation between a cubic austenite phase and a monoclinic martensite phase, albeit through different pathways. SME, the focus of this work, refers to the ability of a deformed SMA specimen to recover its original shape through the application of heat. This paper details the experimental characterization and analysis of SME during the cyclic thermo-mechanical loading of nickel-titanium, also referred to as $\mathrm{NiTi}$ and Nitinol. Nitinol is a widely used SMA due to its advantageous characteristics, including its large actuation stroke of up to $8 \%$ and its high power output.

The SME in Nitinol is achieved through a phase transformation between an austenite (B2 cubic) and martensite (monoclinic) phase, which is schematically shown in Fig. 1 and proceeds as follows: at room temperature, the specimen is primarily comprised of a twinned martensite (B19') phase. When deformation is applied, it is accommodated by the detwinning of this martensite phase. Post-deformation, if the material is then heated above a temperature known as the austenite finish temperature $\left(A_{\mathrm{f}}\right)$, the undeformed specimen will transform to the austenite phase and the material will revert back to its pre-deformed shape. When the specimen is subsequently cooled below a temperature known as the martensite finish temperature $\left(M_{\mathrm{f}}\right)$, the austenite transforms back to a self-accommodated twinned martensite (B19') phase. Martensitic self-accommodation upon cooling sustains the shape of the material as it was in the austenite phase, and the specimen shows no apparent change in shape. This process can be repeated 


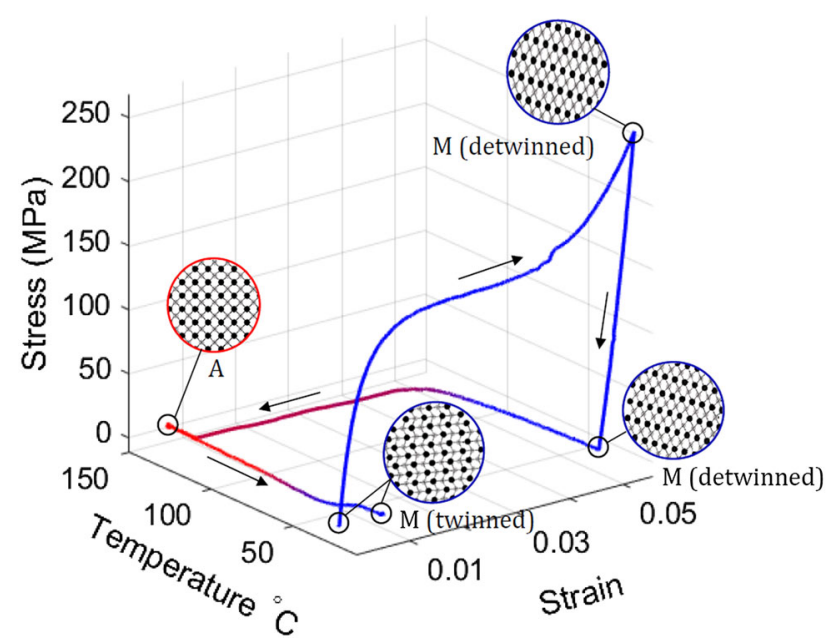

Fig. 1 The stress-strain temperature path followed during the shapememory actuation of nickel-titanium. At lower temperatures (blue region), twinned martensite detwins upon application of mechanical load. Some deformation is recovered upon mechanical unloading wherein the martensite remains detwinned. Subsequently, the temperature is increased, and the detwinned martensite $(\mathrm{M})$ reverts to austenite (A). Finally, there is a transformation from austenite (A) to twinned martensite (M) upon cooling, accompanied by a small amount of residual strain (Color figure online)

through numerous cycles, though significant residual strain can accumulate depending on the severity of thermo-mechanical cycling and the number of cycles performed.

SME is most commonly utilized for actuation with limited space and weight allowances, as is found in automotive, robotics, and aerospace applications. The nature of these applications also requires the actuation to be reliable and fatigue resistant. Although Nitinol is utilized in industrial actuation applications, there is still much that is unknown about the shape-memory effect under cyclic loading, particularly as compared with the superelastic (SE) effect under cycling loading. It is known that several factors can influence the behavior of SE nickel-titanium under cyclic loads, including Ni content, heat treatment of the sample prior to testing, temperature, and strain rate at which the test is conducted, and initial microstructure, among others. Several aspects of the response of SE NiTi under mechanical cycling can be related to the development of dislocations and/or retention of martensitic nuclei, and the interplay between plastic slip and propensity to transform based on crystallographic texture [1-12]. In comparison, the relative lack of information on the SME response under thermo-mechanical cycling is due to, among other factors, experimental difficulties in obtaining the microstructure of martensitic NiTi and in characterizing the self-accommodation process that underlies the shapememory effect.

The size dependence of the SME at the micro- and nanolength scales has come under rapidly increasing interest, both from modeling and experimental perspectives, due to the increases in functionality that can be gained by the implementation of active materials for actuation and energy dissipation in small-scale devices. In this work, transformation characteristics in $500 \mu \mathrm{m}$ diameter $\mathrm{NiTi}$ wires were examined under SME cycling. High-resolution microscale strains were captured using an emerging experimental approach using self-assembled nanoparticles, and used to infer the extent of transformation and examine its spatial similarity with thermo-mechanical cycling. It has been shown in previous work [12-14] that strain can be successfully used to determine transformation location and extent at both the macroscale and the microscale in superelastic Nitinol. At the microscale, full-field strain maps and their relationship to the underlying microstructure were successfully analyzed in superelastic Nitinol in order to determine active variants and the connection between detwinning and residual strain accumulation [14]. In this work on the SME response of Nitinol wires, it was found that the detwinning process was quite heterogeneous at the microscale, and could display a large degree of similarity with thermo-mechanical cycling that increased as cycling progressed.

\section{Background}

There are limited studies on phase transformation during the SME of Nitinol, and these have largely focused on either the macroscale or sub-micrometer length scales. At the macroscopic length scale, global measures like the stress-strain relation are used to improve prediction capabilities. These studies provide valuable information, but are averaged measures that cannot capture the transformation heterogeneity inherent to these materials, nor the dependence of this heterogeneity on the underlying microstructure. As such, an opportunity to control material performance through targeted processing of the microstructure is lost. At sub-micrometer length scales, studies have provided important information about lattice structure, precipitation, and dislocation activity, and have significantly improved our understanding of the underlying transformation mechanisms behind both the SME and SE effects. This length scale captures fundamental parameters of transformation, but the small field of view precludes the mesoscale analysis of the spatial evolution of transformation and its relation to microstructure.

At the macroscopic length scale, SMA wire actuators are subjected to thermo-mechanical cycling during their working life, accumulating plastic deformation. This accumulation of plastic deformation with each cycle is attributed to changes in the microstructure of the wire, whereby lattice defects, dislocations, and precipitates are 
introduced into the matrix and cause significant changes in the capability of the actuator to recover deformation $[15,16]$. It is macroscopically evident that residual strain accumulates through thermo-mechanical cycling in SME (for example see [17-20] and the references contained therein), but the extent of detwinning and accumulation of residual strain at the microstructural length scale in polycrystalline SMAs remains largely uncharacterized.

Sub-micron investigations of SME actuation and residual strain accumulation have focused on the role of martensitic variants and twins, and their interactions with plastic deformation mechanisms. TEM studies of the thermal cycling of NiTi have shown a significant increase in dislocation density [21]. Whether dislocation substructures assist or impede phase transformation is under active debate in the SMA community, but it is clear that they play a significant and important role in determining transformation properties and alloy performance. Simon et al. [22] comprehensively described the need to understand the role of dislocations and dislocation substructures in impeding martensitic transformation during thermal cycling, yet promoting the stress-induced formation of martensite during superelastic cycling, a distinction that is not yet understood. A number of studies indicate that the formation of a dislocation substructure in a SMA, whether through martensitic transformation or plastic deformation of the austenite phase, can act as an obstacle to martensite formation and thereby influence shape recovery [23-25]. Martensites with an internal structure consisting of either twinning (as in NiTi) or stacking faults can allow shape recovery, whereas martensites that are truly slipped internally preclude SME [24, 25]. Kajiwara [25] proposed that the shape strain of the nucleating martensite must be accommodated by plastic deformation in the austenite, and therefore any factors that impede this accommodation, including a lack of dislocations, work hardening, or grain refinement, will suppress transformation. Interestingly, it has also been proposed that dislocations can promote the nucleation, formation, and stabilization of martensite, acting as microstructural markers which allow the material to remember its previous stress-strain history [26, 27]. Additionally, there have been direct TEM observations of martensite nucleation on dislocations on SMAs, including $\mathrm{Cu}-\mathrm{Al}-\mathrm{Ni}$ under superelastic cycling [28] and nucleation of the $R$-phase in NiTi [29].

The shape-memory effect has been observed in a range of small-scale geometries including nanopillars, nanoscale imprints [30], thin films [31, 32], and microwires, with a varied recovery extent based on considerations including geometry and microstructure. For example, complete shape-memory recovery has been observed in tests on micro/nano pillars of a $\mathrm{Cu}-\mathrm{Al}-\mathrm{Ni}$ shape-memory alloy [33]. The authors attribute previously reported problems for martensite nucleation at this scale to a lack in local crystallinity of the sample or, particularly in the case of $\mathrm{NiTi}$, to local deviations in stoichiometry due to titanium depletion from oxidation, in agreement with recent TEM studies on NiTi thin films [34]. It has also been found, on a modeling study of NiFeGa micropillars, that the influence of micropillar aspect ratio and taper angle on the compression response was significantly smaller than the effect of the micropillar diameter [35]. In a study on the shapememory actuation of $\mathrm{Ni}-\mathrm{Mn}-\mathrm{Ga}$ double-beam nanostructures, the SME was observed down to a feature size of $100 \mathrm{~nm}$, behavior different from that observed in constrained geometries like films and nanocrystalline shapememory alloys [36]. Work on CuAlMnNi microwires of 45-255 $\mu \mathrm{m}$ diameter with a bamboo grain structure demonstrated enhanced thermal actuation of lightly biased SMA wires in finer wires; this was attributed to both a decrease in the number of interacting martensite variants as well as to relaxation on the free surface [37].

\section{Materials and Experimental Procedure}

In this study, NiTi wires of $500 \mu \mathrm{m}$ diameter were thermomechanically loaded through the SME cycle in situ in a scanning electron microscope (SEM), and custom deformation tracking methodologies were used to generate fullfield Lagrangian strain maps at the microscale. The wires were 49.5 at.\% $\mathrm{Ni}$ (SM495, black oxide finish, straight wire) manufactured by Nitinol Devices and Components with a $500 \mu \mathrm{m}$ outer diameter and an austenite finish temperature of $84 \pm 5{ }^{\circ} \mathrm{C}$ as determined by differential scanning calorimetry. The grain size was measured to be 20-40 nm using peak widening of the XRD spectrum (Rigaku rotating anode X-ray diffractometer). The diffraction spectrum was obtained at $300{ }^{\circ} \mathrm{C}$ and the grain size was measured using the Scherrer equation (MDI JADE 2010). The wires were cut into sections of $5 \pm 0.1 \mathrm{~cm}$ length with a low-speed diamond saw (South Bay Technologies, Model \#650). To hold the wire samples in place during polishing, they were mounted onto a cylindrical aluminum block with Crystalbond 509-3 (Ted Pella, Inc). The samples were mechanically polished with a Buehler (EcoMet 250) mechanical polisher using grits of 600 (Buehler CarbiMet 2), 800 (Buehler MicroCut Discs), and 1200 (Buehler MicroCut Discs). Following the 1200 grit, the wires were vibratory polished (Pace Technologies GIGA-0900) for $12 \mathrm{~h}$ using non-crystallizing colloidal silica solution (Buehler MasterMet 2, Non-Crystallizing Colloidal Silica Polishing Suspension, particle size $<1$ $\mu \mathrm{m})$. The polishing technique created flat substrate surfaces along the longitudinal axis of the wires for later displacement mapping via nanoparticle tracking. Platinum fiducial 
markers were deposited onto the flat surfaces to mark areas of interest, using focused ion beam (FIB) deposition in an FEI Quanta 3D SEM.

Distortion-corrected digital image correlation (DIC) combined with SEM, termed here as SEM-DIC, was used to quantify high-resolution, full-field strains in fields of view of tens of microns. DIC is a non-contact, full-field surface displacement measurement technique, wherein a random pattern is applied to the specimen surface and tracked as the specimen is deformed. Deformations were calculated by minimizing correlation functions between subsets of the applied pattern, providing a high degree of spatial resolution in the resulting strain fields. As the method is length-scale independent and solely depends on the tracking of the applied pattern, it can be applied to microscale fields of view with no theoretical loss in accuracy; however, in practice, the use of DIC with SEM introduces a number of distortion and noise error sources that require correction [38-40].

For the microscale tracking in this work, an assembly of gold nanoparticles on a chemically functionalized surface was self-assembled following the procedure outlined in [41] for deformation tracking. The gold nanoparticles were fabricated following the Frens technique [42] at an optimal size dictated by the field of view, image resolution, and the roughly $3 \times 3$ rule of thumb for digital image correlation [43]. The size of the nanoparticles can be manipulated down to $20 \mathrm{~nm}$ through the addition of sodium citrate, as determined by Frens [42], whose data the authors plotted in Fig. 2 to facilitate nanoparticle fabrication of the correct size. The sample surface was pretreated in $100{ }^{\circ} \mathrm{C}$ air in the box furnace in order to promote the growth of a layer of $\mathrm{TiO}_{2}$ and subsequent hydroxide (-OH) group to serve as a substrate surface to which silane molecules could attach. The samples were then soaked in a methanol 4:1 APTMS to add a coat of silane molecules to the hydroxide groups, cleaned with methanol to remove excess silane molecules, and soaked in the suspension of gold nanoparticles to deposit the speckle pattern. The silane molecules served as adhesive between substrate surface and the gold nanoparticles, resulting in a random, isotropic pattern at a controlled density and size for high-resolution microscale deformation tracking. An example of a nanoparticle selfassembly on a NiTi wire specimen is shown in Fig. 3.

To obtain microscopic data, the specimens were tested in the experimental setup shown in Fig. 4, with the specimen geometry and test field of view shown schematically in Fig. 5. The wires were loaded in-SEM to a globally applied axial strain of 0.06 using displacement control at a strain rate of $10^{-4} \mathrm{~s}^{-1}$. After reaching a globally applied axial strain of 0.06 , the sample was unloaded until the stage load cell read $0 \mathrm{~N}$, then taken out of the tensile tester and heated to $200 \pm 2.3{ }^{\circ} \mathrm{C}$ (K-type thermocouple, Omega) to

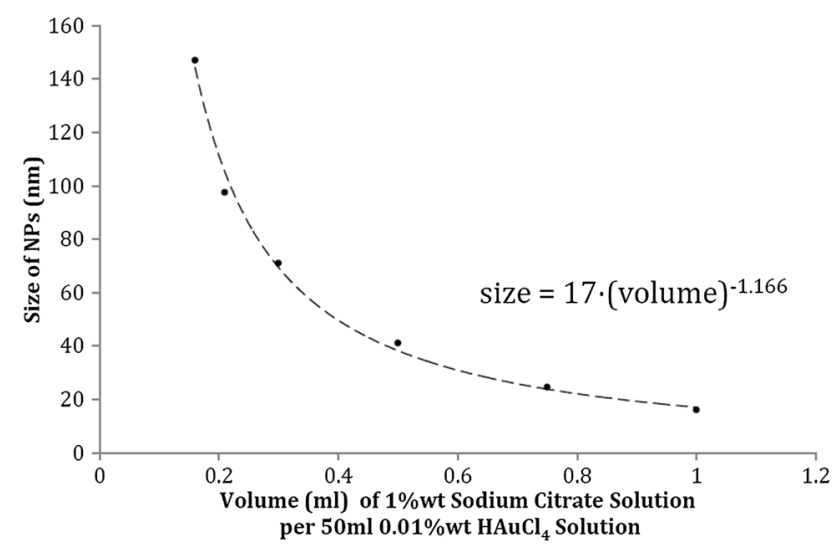

Fig. 2 Nanoparticle size is controlled by the ratio between sodium citrate and $\mathrm{HAuCl}_{4}$. Data points originally reported by Frens [42] are plotted and fit with an exponential relationship for ease of nanoparticle fabrication of the appropriate size

allow strain recovery. SEM images of the area of interest were acquired at steps of $100 \mu \mathrm{m}$ during loading, and after strain recovery when the sample was heated above $A_{\mathrm{f}}$. In order to acquire SEM images, the specimen was held at constant displacement for approximately $10 \mathrm{~min}$ at each of these steps to allow for relaxation and thus prevent image shifting in the field of view. Microscale testing was performed in a Tescan MIRA3 GMH SEM, with a spot size of $7 \mathrm{~nm}$, an image size of $70 \mu \mathrm{m} \times 70 \mu \mathrm{m}$, pixel resolution of $2048 \times 2048$ pixels, scan speed of 4 (dwell time $3.2 \mu \mathrm{sec}$ ), pixel size of $34.2 \mathrm{~nm}$, beam current of $187 \mu \mathrm{A}$, voltage of $10 \mathrm{keV}$, and a $2 \times$ frame integration. Images were collected at $70 \mu \mathrm{m} \times 70 \mu \mathrm{m}$ to optimize nanoparticle size to pixel

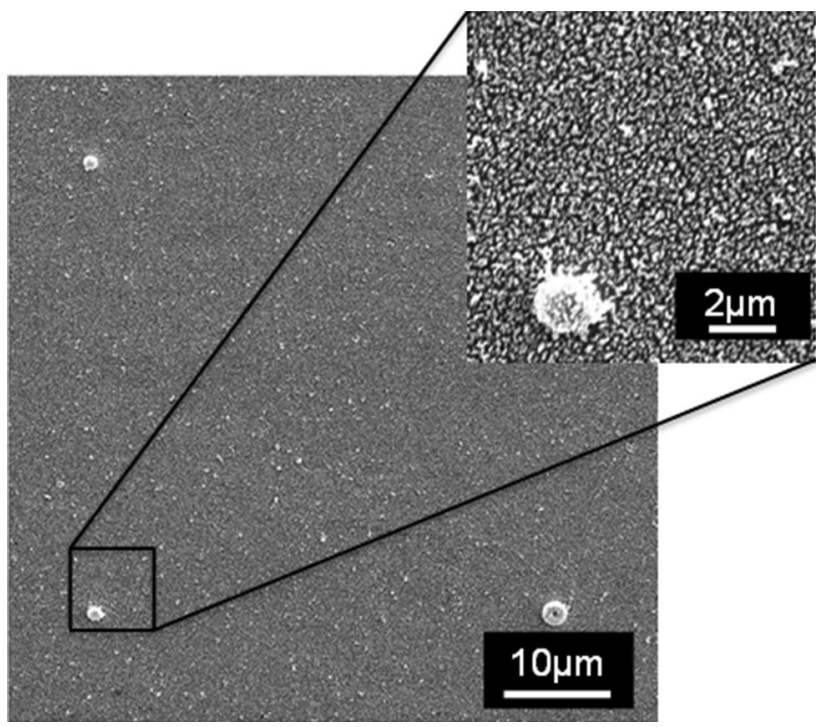

Fig. 3 An example of a self-assembly of nanoparticles comprised of approximately $100 \mathrm{~nm}$ diameter gold nanoparticles, used for microscale deformation mapping via SEM-DIC. The larger white dots at the four corners of the field of view are platinum markers that were FIBdeposited to designate the field of view 

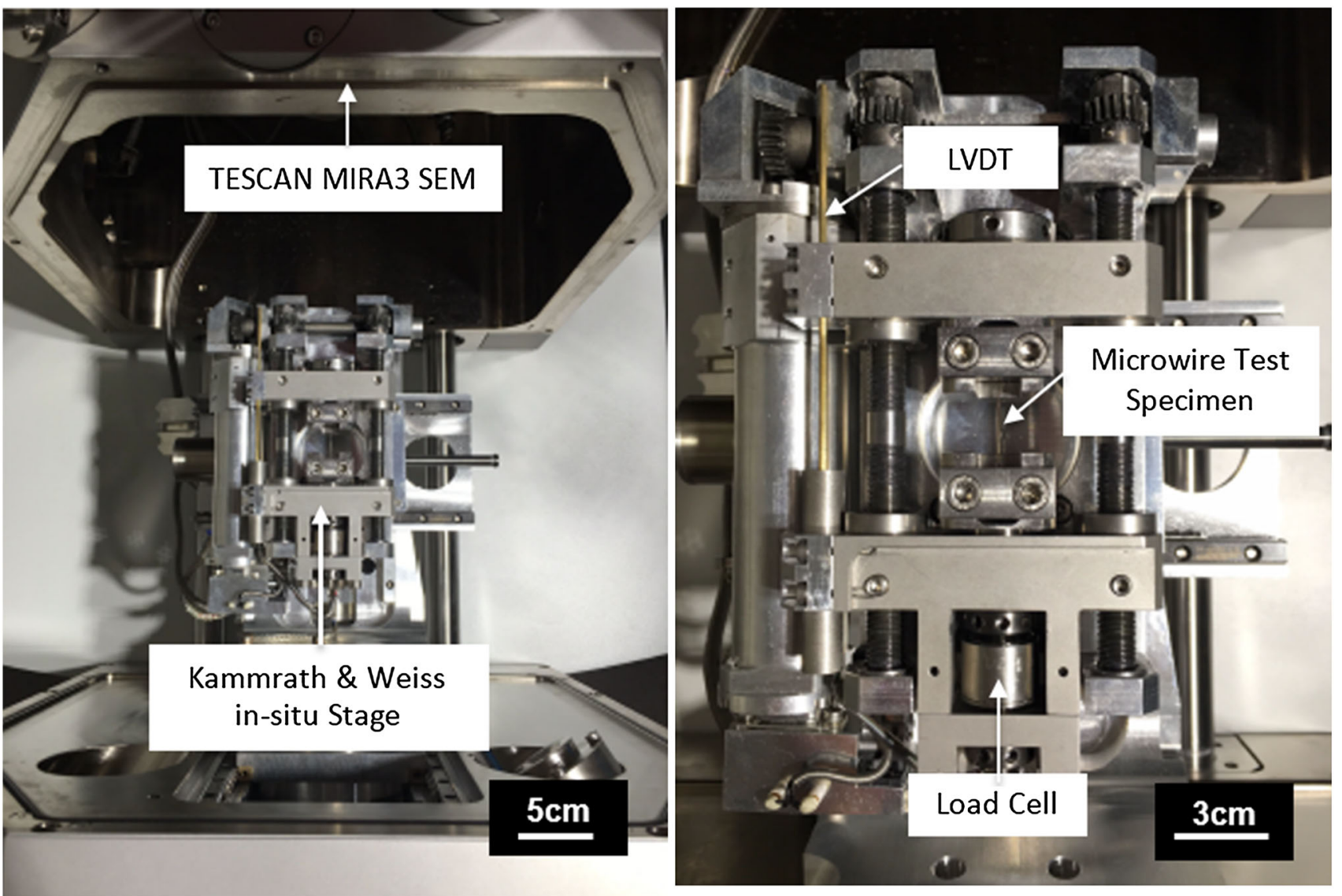

Fig. 4 In-SEM testing was performed using a tensile stage (Kammrath and Weiss) with a $10 \mathrm{lb}$ tension-compression load cell (Honeywell model\# 3108-10) in the scanning electron microscope (Tescan MIRA3) shown above

Fig. 5 Schematic of the microwire specimen geometry, test field of view, and uniaxial loading condition. The $34 \mu \mathrm{m} \times$ $34 \mu \mathrm{m}$ field of view was located on the flat surface as illustrated. Gage length represents the distance between the crossheads of the in situ load frame, measured by caliper with a resolution $0.03 \mathrm{~mm}$

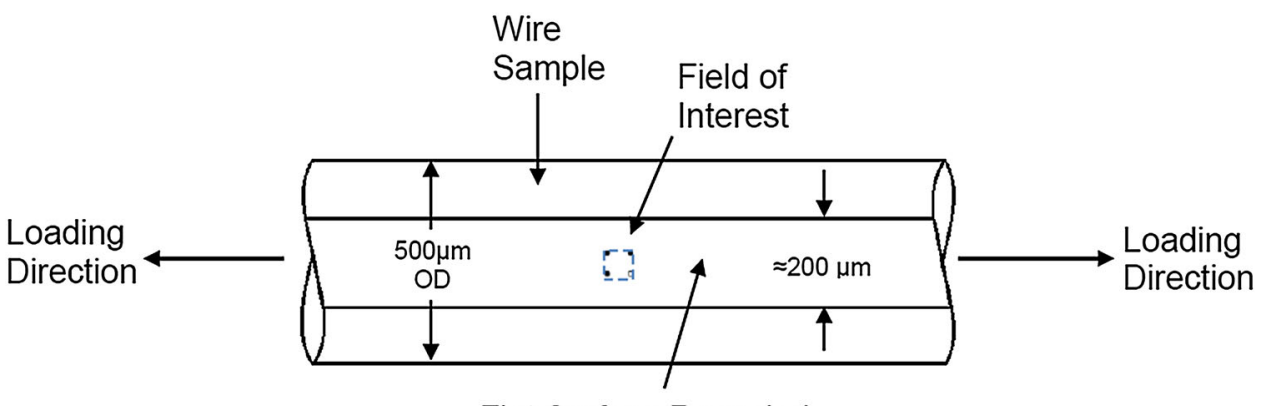

Flat Surface Revealed by Mechanical Polishing

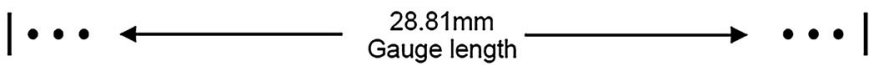

ratio, and later reduced to $34 \mu \mathrm{m} \times 34 \mu \mathrm{m}$ for analysis on the region with best nanoparticle pattern. The location of data collection on the specimen is shown schematically in Fig. 5. The captured micrographs were first processed by commercial digital image correlation software [44], following which spatial and time-dependent distortions inherent to SEM imaging were corrected. Validation of and procedures for distortion correction are detailed in [40] and are based on a framework originally developed by Sutton et al. [38, 39]. The undeformed (twinned) martensite was taken as the reference configuration of zero strain. Lagrangian surface strains were calculated using the corrected displacement information. The in-SEM stress-strain responses obtained using the load from the SEM stage load cell, and the global (LVDT measuring grip displacements) and local (DIC-averaged in the FOV) strains, are shown in 
Figs. 6 and 8 and discussed in later sections of the paper. As expected, the global strains are larger, primarily due to a small amount of grip slippage (on the order of $10 \mu \mathrm{m}$ ) of the wires.

\section{Results and Discussion}

\section{Spatial Heterogeneity of Strain During 1st Actuation Cycle}

There was a pronounced heterogeneity in the strain distribution evident at the microscale caused by detwinning of the martensite phase upon application of load. Detwinning is known to be directional with respect to texture, where it is enhanced for certain directions and reduced for others; this is the first direct observation, to the best of the authors' knowledge, of the extent of that anisotropy at the microscopic length scale encompassing several grains. This heterogeneity is shown in Fig. 6, where the principal Lagrangian $\left(\varepsilon_{1}\right)$ strains incurred by martensite detwinning during a full actuation cycle (cycle 1) are shown for a 34 $\mu \mathrm{m} \times 34 \mu \mathrm{m}$ field of view. Points (a-f) in the macroscopic loading curve correspond with the microscale strain maps labeled a-f. Point a, when the specimen is macroscopically considered twinned martensite, is taken as the reference (zero strain) map. All subsequent strain maps in this work also reference the unloaded, room temperature specimen of twinned martensite as the reference/zero strain. The field of view was captured in the approximate horizontal and vertical center of the wire specimen that had a gage length of $28.81 \pm 0.03 \mathrm{~mm}$ as shown in Fig. 5, and contained 990,025 data points $(995 \times 995$ pixels $)$. Each data point was approximately $34 \mathrm{~nm} \times 34 \mathrm{~nm}$; therefore, the magnitude of strain was taken to indicate the relative extent of deformation, including detwinning and plasticity, across an area incorporating a number of martensitic twins. In strain map c, taken at maximum load when there was a large amount of detwinning, there was significant heterogeneity in the degree to which detwinning took place; there are nominally five micron regions that showed little to no incurred strain (denoted in blue), whereas immediately adjacent regions showed strains on the order of 0.08 (denoted in yellow).

The heterogeneous distribution of strains resulting from detwinning remained consistent throughout the SME cycle, although the magnitudes changed. At point $b$, the specimen is nominally midway through the macroscopic detwinning process, and the evolution of the heterogeneous strain field is apparent. At point c, further load has been globally applied, and the strain field now has the same distribution but a greater magnitude of heterogeneity, where local high strain regions from point $b$ intensify. At point $d$, upon unloading, the strain magnitude lessens slightly, but its distribution remains consistent. The amount of strain each data point recovered upon unloading (Fig. 6, from map c to d) showed no dependence on how much strain was initially present at that data point in map c; each data point reduced by nominally the same amount. Even upon heating the specimen (point e), wherein there is a reversion back to the (primarily) austenite phase and subsequent recovery of the deformation, there remains a faint 'ghost' pattern of the induced strain. Note that maps e and $\mathrm{f}$ in Fig. 6 are at a reduced strain scale of $[-0.02,0.02]$, versus maps a-d at a strain scale of $[-0.05,0.1]$, in order to clearly show the heterogeneity at greatly reduced strains, and thus show more detail in the strain field. At points e and $f$, the strain magnitude is greatly reduced, but the heterogeneity remains the same as in point $\mathrm{c}$, when the specimen was primarily in the detwinned martensite phase at maximum load. When the specimen cools (point f) and macroscopically reverts back to twinned martensite, the ghost pattern seen at point $\mathrm{E}$ from the initial loading and subsequent detwinning remains, with a reduction in averaged strain from point e to point $\mathrm{f}$ (cooling and overall transformation from austenite to twinned martensite) of $0.55-0.39 \%$. The strains at maximum load (map c) were largely recovered upon heating and subsequent cooling of the specimen and its reversion back to twinned martensite, with a small degree of residual strain from locked-in martensite and plasticity remaining, as evident in map $f$ and in the macroscopic stress-strain temperature curves at the top of Fig. 6.

Residual strain concentrated at locations where strain accumulation from detwinning and plasticity were significant, as evident in a comparison between Fig. 6c (where significant stress-induced detwinning has taken place) and Fig. 6f (where the specimen has been heated and cooled, and has reverted back to primarily austenite with a corresponding recovery in shape). Please note that Fig. 6c, f use different strain scale bars in order to highlight the similarity in the pattern of strain; however, the magnitudes of the residual strain in Fig. 6f are much reduced compared to Fig. 6c at maximum load. The persistence of the strain pattern indicates that the process of martensitic detwinning in a SME cycle, and the associated plasticity that occurs with it, is spatially correlated to the subsequent accumulation of residual strain at the microscale. As shown in Fig. 7, the recovered strain (Fig. 6, strain map f subtracted from strain map c) showed a linear dependence with the maximum strain (Fig. 6, strain map c) at each individual data point. In Fig. 7, each of the nearly one million data points in the map is individually plotted. The $z$-axis (normalized count calculated with respect to the total number of data points) indicates the proportion of data points that fall into each strain data bin of $0.0004 \times 0.0004$ in size. 

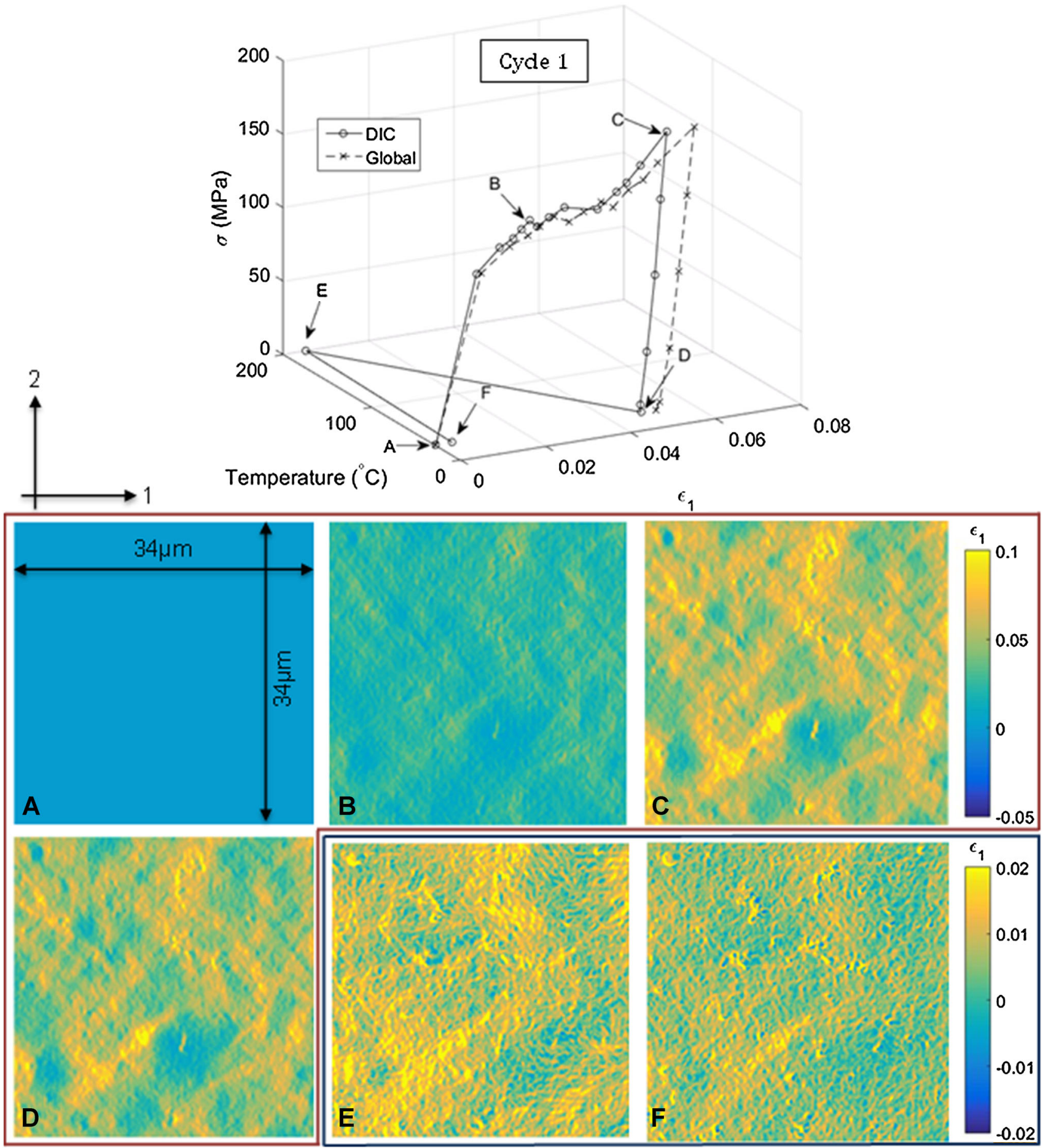

Fig. 6 Microscale strain maps corresponding to the designated points in the stress-strain temperature curve. There was a pronounced heterogeneity in the strain distribution evident at the microscale, caused by detwinning of the martensite phase upon application of load. This heterogeneous strain distribution remained constant throughout the entire actuation cycle, although the strain magnitude changed. Residual strain concentrated at locations where strain accumulation from detwinning and plasticity were significant, as evident in a comparison between Fig. 7c (where significant stressinduced detwinning has taken place) and Fig. $7 \mathrm{f}$ (where the specimen has been heated and cooled, and has reverted back to primarily austenite with a corresponding recovery in shape). Please note the two different scale bars for figures (a-d) and figures $(\mathbf{e}, \mathbf{f})$ 


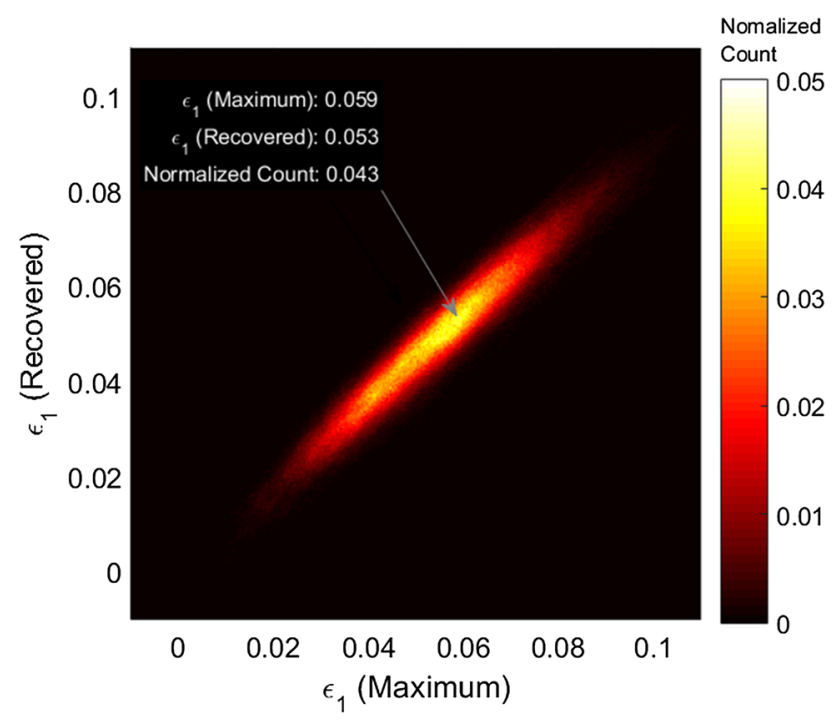

Fig. 7 A heat map of the strain at each data point in cycle 1 is shown, where the color indicates the normalized count of the number of data points with those $x$ - and $y$-values. The $x$-axis is the value of the point at strain map c (detwinned martensite at maximum load in Fig. 6), and the $y$-axis is the value at that point of strain map of $\mathrm{f}$ subtracted from strain map c (where $\mathrm{f}$ is the twinned martensite after heating and subsequent cooling in Fig. 6). Deformation was largely recovered after heating, and the recovered strain between maps $\mathrm{c}$ and $\mathrm{f}$ showed a strong linear dependence on the maximum strain of the detwinned martensite (map c)

Therefore, the grids of recovered strain $[-0.01,0.11]$ and maximum strain $[-0.01,0.11]$ are sectioned into $300 \times$ 300 bins. Significant strain recovery accompanied the stress-induced detwinning at nearly all points in the field of view, as indicated by the linearity of the heat map; for example, the largest number of data points on cycle 1 (constituting $4.3 \%$ of the total number of data points and labeled with the black arrow on Fig. 7) had a maximum strain of 0.059 and a recovered strain of 0.053 .

Despite the local strain heterogeneity, the averaged DIC-calculated strains matched the globally acquired strains well, as shown in the macroscopic stress-strain curve for cycle 1 in Fig. 6 . The global strain was calculated from the displacement of the crossheads of the in-SEM testing stage as measured by LVDT. In both cases, the applied nominal stress was calculated from the applied load measured by the in-SEM stage load cell, divided by the initial cross sectional area of the wire. The DIC and global curves were in reasonable agreement with discrepancy attributed to two factors: one, that the DIC-obtained strains were only captured and averaged in a $34 \mu \mathrm{m}$ field of view, whereas the global strains were taken across the entire $28.81 \pm 0.03 \mathrm{~mm}$ gage section, in a process that is mesoscopically heterogeneous; and two, that due to the practical difficulty of gripping wires for testing, there was likely grip slippage, wherein only $10-20 \mu \mathrm{m}$ of slippage would account for the differences in strain. As one would expect in this case, the global curve shows the larger strain values.

\section{Cycle-to-Cycle Similarity During Subsequent Actuation}

Once established in the first actuation cycle, the same microscale pattern of strain reappeared in subsequent cycles, as shown in Fig. 8. Here, the microscale strains of the (primarily) detwinned martensite at the maximum load (strain maps at point c) and of the (primarily) twinned martensite of the unloaded sample (strain maps at point $f$ ) are shown side by side during the first, second, and fifth actuation cycles. Note that all maps are correlated with respect to the original reference image of the undeformed, twinned specimen as shown in Fig. 6a. Also, for ease of comparison, the strain maps in Fig. 8 are shown with two different scale bars: the maximum strain at map $\mathrm{c}$ is shown with a relatively broad scale bar of $\varepsilon_{1}=[-0.05,0.1]$ and the residual strain at map $\mathrm{f}$ is shown with a scale bar of $\varepsilon_{1}=[-0.02,0.02]$. The same microscale pattern of strain reappeared at both the maximum load and the recovered specimen, with an intensification of the residual strain in map $\mathrm{f}$ from cycle 1 to cycle 5 . The averaged strain over the field of view for both maps increased from cycle 1 to cycle 5 , from a strain of 0.0543 to 0.0561 (map c) and from 0.0039 to 0.0080 (map f), respectively.

Correlation coefficients calculated point-by-point were used to quantify the persistence of microscale strain heterogeneity from cycle-to-cycle, as shown in Fig. 9 for cycle 1 and cycle 2 . Correlation coefficients were calculated when the specimen was at maximum load at a given cycle, i.e., between the strain maps at point $\mathrm{c}$ in Figs. 6 and 8. The magnitude of the strain maps at point $\mathrm{C}$ varied depending on the cycle number, as expected given the small field of view, heterogeneity of transformation, and accumulation of residual strain; however, the strains averaged over the field of view were similar. The maps at point $\mathrm{c}$ in Figs. 6 and 8 were taken at maximum load on the specimen, which was measured as DIC-averaged strain values over the field of view of 0.0543 (cycle 1), 0.545 (cycle 2), and 0.561 (cycle 5). The correlations calculated point-by-point between cycles 1 and 2 are shown in the form of a heat map in Fig. 9. This map shows the number of data points (counts) on the $z$-axis that fall into a certain $x-y$ bin, where $x$ is the correlation coefficient between the cycles at their maximum load (e.g., strain map c in Fig. 6), and $y$ is the maximum strain at that point in the first actuation cycle. Note that the correlation coefficient neglects the effect of strain magnitude, but instead reports the similarity between the trends of two datasets. The correlation coefficient at a data point $(x, y)$ represents the linear dependence of the strain at that point at the two 

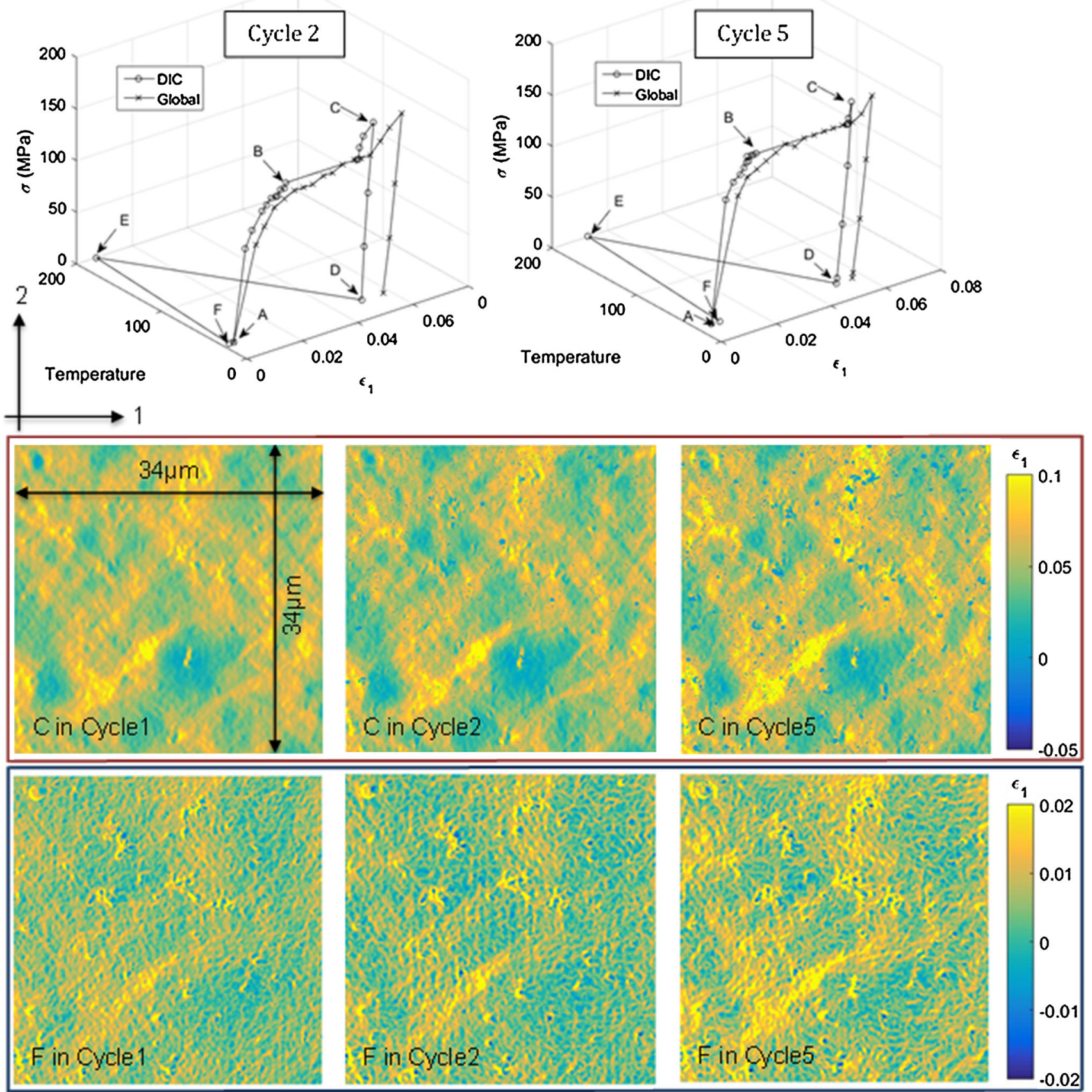

Fig. 8 The strain maps acquired in the detwinned martensite at maximum applied load (map c) as well as in the twinned martensite after mechanical unloading, heating, and cooling (map f). The same microscale pattern of strain reappeared at both the maximum load and the unloaded specimen, with an intensification of the residual strain in

cycles under comparison, and ranges between -1 and +1 . A correlation coefficient approaching +1 indicates that the strains are strongly positively correlated, and a correlation coefficient of 0 indicates that the strains have no correlation. The $z$-axis (normalized count) indicates how many data points fall into each data bin of 0.00125 (strain) by map $\mathbf{f}$ from cycle 1 to cycle 5. The averaged strain over the field of view for both maps increased from cycle 1 to cycle 5 , from a strain of 0.0543 to 0.0561 (map c) and from 0.0039 to 0.0080 (map f), respectively

0.01 (correlation coefficient) in size. Therefore, the grid of correlation coefficient $[-1,1]$ and strain $[0,0.15]$ are sectioned into $200 \times 120$ bins.

The two maximum strain maps (c) have strong point-topoint similarity between cycles 1 and 2 , as observed in Fig. 8 and graphically illustrated in Fig. 9. Figure 9 shows 


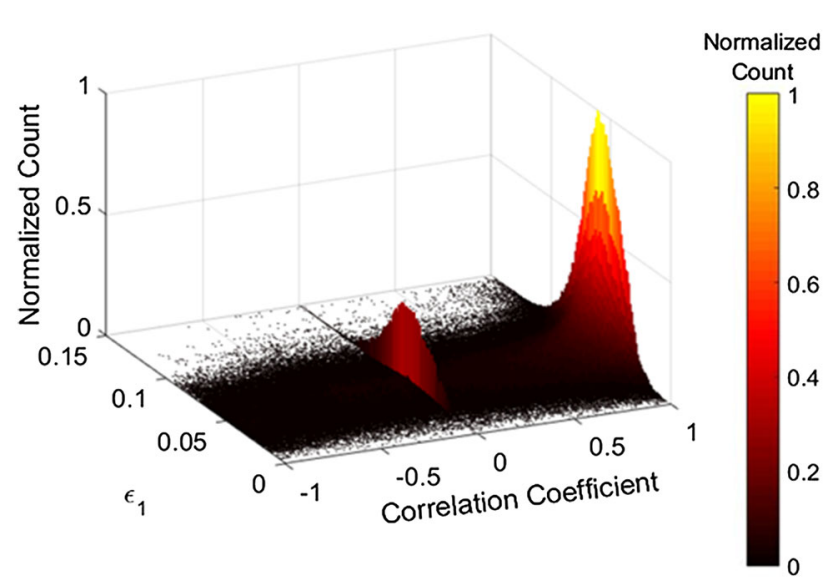

Fig. 9 A heat map of the point-by-point correlation coefficients between microscale strain maps $\mathbf{c}$ for cycles 1 and 2 (in Fig. 8). There is a strong point-to-point similarity between cycles 1 and 2 , as observed in Fig. 8 and tabulated here. The "Correlation Coefficient" axis refers to the correlation coefficient between the strains at each selected data point in cycles 1 and 2 , and the " $\varepsilon_{1}$ " axis refers to the strain at that same data point in cycle 1 . The $z$-axis, "Normalized Count," tabulates the number of pixels that fall into each of these axes values. A small number of points that failed digital image correlation, or did not show a correlation to each other, constitute a short peak around a correlation coefficient of 0

a heat map of the point-by-point correlation coefficients between microscale strain maps $\mathrm{c}$ for cycles 1 and 2 (shown in Fig. 8), which were taken at maximum DICaveraged strains of 0.0543 (cycle 1) and 0.0545 (cycle 2). The "Correlation Coefficient" axis refers to the correlation coefficient between the strains at each selected data point in cycles 1 and 2 , and the " $\varepsilon_{1}$ " axis refers to the strain at that same data point in cycle 1 . The $z$-axis, "Normalized Count," tabulates the number of pixels that fall into each of these axes values. The majority of the correlation coefficient values in the map belong to a large peak close to 1 , indicating a very strong similarity of the maps for most data points. In this cluster of data points, $51.9 \%$ of the data points have a 'strong' correlation coefficient (greater than 0.8 ), $40.4 \%$ of the data points have a correlation coefficient greater than 0.9 , and $29.4 \%$ have a correlation coefficient greater than 0.95 . A small number of points $(2.7 \%$ of the total number of points) that failed digital image correlation constitute a short peak around a correlation coefficient of 0 . When the points that failed digital image correlation were excluded from this plot, the peak disappeared.

Correlation coefficients between each individual data point tended to increase with thermo-mechanical cycling. For example, in cycle 1 versus cycle 2 as shown in Fig. 9, $40.4 \%$ of data points had a correlation coefficient greater than 0.9 , and $29.4 \%$ of data points had a correlation coefficient greater than 0.95 . The percentage of data points with high correlation coefficients increased between cycles 4 and 5 , where $44.2 \%$ of data points had a correlation coefficient greater than 0.9 , and $32.5 \%$ of data points had a correlation coefficient greater than 0.95 . This shift of distribution towards higher percentages of strong correlation coefficients indicates that there is a 'settling in' process, where the similarity of strain induced by detwinning and plasticity becomes stronger as cycling progresses. This also indicates that, if strain accommodation can be controlled in the first actuation cycle by the appropriate microstructural design, then this control can carry through subsequent cycles for potentially the lifetime of the shape-memory component. Future work is needed to investigate the relationship between detwinning and microstructural landscape, and the effect of microstructural and material characteristics on the persistence of accommodated strain with repeated shape-memory actuation.

\section{Conclusions}

In this work, the microscale transformation characteristics of Nitinol wires of $500 \mu \mathrm{m}$ diameter were examined during shape-memory actuation cycles via a novel method combining scanning electron microscopy, distortion-corrected digital image correlation, and the self-assembly of nanoparticles on the specimen surface through chemical functionalization. These tests resulted in the following findings:

- The work provides, to the best of the authors' knowledge, the first direct observation of full- field strain distribution at the microscale of SME NiTi during thermo-mechanical cycling.

- There was a pronounced heterogeneity in the strain distribution evident at the microscale caused by martensitic detwinning upon load application. At maximum load when there was a large amount of detwinning, there was significant heterogeneity in the strains, which is indicative of the degree to which detwinning took place; there were micron-sized regions (encompassing numerous grains with a grain size of 20-40 nm) that showed little to no incurred strain, whereas immediately adjacent regions showed strains on the order of 0.08 .

- The heterogeneous distribution of strain (as referenced back to a zero strain configuration of the twinned martensitic sample at the beginning of cycle 1) remained consistent throughout the SME cycle, although the magnitudes of strain changed. The amount of strain each data point recovered upon unloading (Fig. 6, from map $\mathrm{c}$ to d) showed no dependence on how much strain was initially present at that data point at maximum load in cycle 1 (map c); each data point reduced by nominally the same amount. 
- Residual strain concentrated at locations where strain accumulation from detwinning and plasticity were significant. The persistence of the strain pattern indicates that the process of martensitic detwinning in a SME cycle, and the associated plasticity that occurs with it, is spatially correlated to the subsequent accumulation of residual strain at the microscale.

- In addition to persisting throughout a single cycle, the same microscale pattern of strain reappeared in subsequent actuation cycles as evident in Fig. 8.

- Correlation coefficients between each individual data point tended to increase with thermo-mechanical cycling. For example, in cycle 1 versus cycle 2 as shown in Fig. 9, 40.4\% of data points had a correlation coefficient greater than 0.9 , and $29.4 \%$ of data points had a correlation coefficient greater than 0.95 . The percentage of data points with high correlation coefficients increased between cycles 4 and 5, where $44.2 \%$ of data points had a correlation coefficient greater than 0.9 , and $32.5 \%$ of data points had a correlation coefficient greater than 0.95 . This shift of distribution towards higher percentages of strong correlation coefficients indicates that there is a 'settling in' process, where the similarity of strain induced by detwinning and plasticity becomes stronger as cycling progresses.

Acknowledgements The authors gratefully acknowledge the financial support for this work, provided by General Motors Company through the GM/UM Smart Materials and Structures Collaborative Research Laboratory. The authors would also like to acknowledge helpful discussions with, and experimental assistance from, Prof. John Shaw and Prof. Diann Brei, Michael Kimiecik, Will LePage, and Daniel Biggs at the University of Michigan.

\section{References}

1. Eggeler G, Hornbogen E, Yawny A, Heckmann A, Wagner M (2004) Structural and functional fatigue of NiTi shape memory alloys. Mater Sci Eng 378(1-2):24-33

2. Gall K, Maier HJ (2002) Cyclic deformation mechanisms in precipitated NiTi shape memory alloys. Acta Mater 50:4643-4657

3. Iadicola MA, Shaw J (2002) The effect of uniaxial cyclic deformation on the evolution of phase transformation fronts in pseudoelastic NiTi wire. J Intel Mater Syst Struct 13(2/ 3): $143-156$

4. Lim TJ, McDowell DL (1994) Degradation of an NiTi alloy during cyclic loading. In: Proceedings of the North American conference on smart structures and materials. SPIE, 153-165

5. Matsuzaki Y, Naito H, Ikeda T, Funami K (2001) Thermomechanical behavior associated with pseudoelastic transformation of shape memory alloys. Smart Mater Struct 10(5):884-892

6. McCormick PG, Liu Y (1994) Thermodynamic analysis of the martensitic transformation in NiTi-II. Effect of transformation cycling. Acta Metall Mater 42(7):2407-2413

7. Melton KN, Mercier O (1979) Fatigue of NiTi thermoelastic martensites. Acta Metall 27(1):137-144
8. Shaw JA, Kyriakides S (1995) Thermomechanical aspects of NiTi. J Mech Phys Solids 42(8):1243-1281

9. Shaw JA, Kyriakides S (1997) On the nucleation and propagation of phase transformation fronts in a NiTi alloy. Acta Mater 45(2):683-700

10. Strnadel B, Ohashi S, Ohtsuka H, Ishihara T, Miyazaki S (1995) Cyclic stress-strain characteristics of $\mathrm{Ti}-\mathrm{Ni}$ and $\mathrm{Ti}-\mathrm{Ni}-\mathrm{Cu}$ shape memory alloys. Mater Sci Eng 202:148-156

11. Strnadel B, Ohashi S, Ohtsuka H, Miyazaki S, Ishihara T (1995) Effect of mechanical cycling on the pseudoelasticity characteristics of Ti-Ni and Ti-Ni-Cu alloys. Mater Sci Eng 203:187-196

12. Kim K, Daly S (2011) Martensite strain memory in the shape memory alloy nickel-titanium under mechanical cycling. Exp Mech 51:641-652

13. Kimiecik M, Jones JW, Daly S (2015) Grain orientation dependence of martensitic phase transformation in polycrystalline shape memory alloys. Acta Mater 94:214-223

14. Kimiecik M, Jones JW, Daly S (2016) The effect of microstructure on stress-induced martensitic transformation under cyclic loading in the SMA nickel-titanium. J Mech Phys Solids 89:16-30

15. Wagner MFX, Dey SR, Gugel H, Frenzel J, Somsen Ch, Eggeler $G$ (2010) Effect of low temperature precipitation on the transformation characteristics of Ni-rich NiTi shape memory alloys during thermal cycling. Intermetallics 18:1172-1179

16. Ibarra A, San Juan J, Bocanegra EH, Nò ML (2007) Evolution of microstructure and thermomechanical properties during superelastic compression cycling in $\mathrm{Cu}-\mathrm{Al}-\mathrm{Ni}$ single crystal. Acta Mater 55:4789-4798

17. Bhattacharya K (2003) Microstructure of martensite: why it forms and how it gives rise to the shape memory effect, 1 st edn. Oxford University, Oxford

18. Churchill CB (2010) Experimental techniques for characterizing the thermo-electro-mechanical shakedown response of SMA wires and tubes. Ph.D. Thesis, University of Michigan Library, USA

19. Bertacchini O, Mabe J, Lagoudas D, Calkins F (2008) Thermomechanical cyclic loading and fatigue life characterization of nickel rich NiTi shape memory alloy actuators. In: Proceedings of SPIE, 6929

20. Hebda DA, White SR (1995) Effect of training conditions and extended thermal cycling on nitinol two-way shape memory behavior. Smart Mater Struct 4(4):298

21. Pelton AR, Huang GH, Moine P, Sinclair R (2012) Effect of thermal cycling on microstructure and properties in nitinol. Mater Sci Eng 532:130-138

22. Simon T, Kroger A, Somsen C, Dlouhy A, Eggeler G (2010) On the multiplications of dislocations during martensitic transformations in NiTi shape memory alloys. Acta Mater 58:8150

23. Perkins J (1974) Martensitic substructural prerequisites for shape memory effect (SME) behavior. Scr Metall 9:121-128

24. Otsuka K, Wayman CM (1975) On the shape memory effect in internally faulted martensites. Scr Metall 9:1017-1022

25. Kajiwara S (1986) Roles of dislocations and grain boundaries in martensite nucleation. Metall Trans A 17(10):1693-1702

26. Knapp H, Dehlinger U (1956) Mechanics and kinetics of martensite formation without diffusion. Acta Metall 4:289

27. Yawny A, Sade M, Eggeler G (2005) Pseudoelastic cycling of ultra-fine-grained NiTi shape-memory wires. Z Metallkd 96:608

28. Ibarra A, Caillard D, San Jaun J, No ML (2007) Martensite nucleation on dislocations in $\mathrm{Cu}-\mathrm{Al}-\mathrm{Ni}$ shape memory alloys. Appl Phys Lett 90(10):101907

29. Fukuda T, Saburi T, Doi K, Nenno S (1992) Nucleation and selfaccommodation of the R-phase in Ti-Ni alloys. Mater Trans 33(3):271-277 
30. Frick CP, Lang TW, Spark K, Gall K (2006) Stress-induced martensitic transformations and shape memory at nanometer scales. Acta Mater 54(8):2223-2234

31. Busch JD, Johnson AD, Lee Ch, Stevenson DA (1990) Shapememory properties in Ni-Ti sputter-deposited film. J Appl Phys 68(12):6224-6228

32. Shaw GA, Stone DS, Johnson AD, Ellie AB, Crone WC (2003) Shape memory effect in nanoindentation of nickel-titanium thin films. Appl Phys Lett 83:257

33. San Juan J, No ML, Schuh CA (2008) Superelasticity and shape memory in micro- and nanometer-scale pillars. Adv Mater 20(2):272-278

34. Li HX, Mao SC, Zang KT, Liu Y, Guo ZW, Wang SB, Zhang YF, Yin XQ (2014) An in situ TEM study of the size effect on the thermally induced martensitic transformation in nanoscale NiTi shape memory alloy. J Alloy Compd 588:337-342

35. Hernandez E, Lagoudas D (2015) Modeling size effects on the transformation behavior of shape memory alloy micropillars. J Micromech Microeng 25(7):075001

36. Kohl M, Schmitt M, Backen A, Schultz L, Krevet B, Fahler S (2014) Ni-Mn-Ga shape memory nanoactuation. Appl Phys Lett 104(4):043111

37. Tuncer N, Qiao L, Radovitzky R, Schuh C (2015) Thermally induced martensitic transformations in $\mathrm{Cu}$-based shape memory alloy microwires. J Mater Sci 50(22):7473-7487
38. Sutton MA, Li N, Joy DC, Reynolds AP, Li X (2007) Scanning electron microscopy for quantitative small and large deformation measurements. Part I: SEM imaging at magnifications from 200 to 10,000. Exp Mech 47:775-787

39. Sutton MA, Li N, Garcia D, Cornille N, Oretu JJ, McNeill SR, Schreier HW, Li X, Reynolds AP (2007) Scanning electron microscopy for quantitative small and large deformation measurements. Part II: experimental validation for magnifications from 200 to 10,000. Exp Mech 47:789-804

40. Kammers AD, Daly S (2013) Digital image correlation under scanning electron microscopy: methodology and validation. Exp Mech 53(9):1743-1761

41. Kammers AD, Daly S (2013) Self-assembled nanoparticle surface patterning for digital image correlation in a scanning electron microscope. Exp Mech 53(8):1333-1341

42. Frens G (1973) Controlled nucleation for the regulation of the particle size in monodisperse gold suspensions. Nat Phys Sci 241:20-22

43. Sutton MA, Orteu JJ, Schreier HW (2009) Digital image correlation. Image correlation for shape, motion and deformation measurements, 1st edn. Springer, New Mexico

44. Vic-2D 2009, Correlated Solutions Inc., Columbia, South Carolina, USA 